\title{
Germanica
}

\section{La réécriture de Bouvard et Pécuchet dans Un célibataire entre deux âges (1915), Recherches d'un chien (1922) de Franz Kafka et dans Toute une histoire (1995) de Günter Grass}

Die Umarbeitung von Bouvard et Pécuchet in Un célibataire entre deux âges (1915), Recherches d'un chien (1922) von Franz Kafka und in Toute une histoire (1995) von Günter Grass.

Florence Bancaud

\section{OpenEdition}

\section{Journals}

Édition électronique

URL : http://journals.openedition.org/germanica/2068

DOI : 10.4000/germanica.2068

ISSN : 2107-0784

Éditeur

Université de Lille

Édition imprimée

Date de publication : 31 décembre 2002

Pagination : 39-58

ISBN : 9782913857100

ISSN : 0984-2632

\section{Référence électronique}

Florence Bancaud, «La réécriture de Bouvard et Pécuchet dans Un célibataire entre deux âges (1915),

Recherches d'un chien (1922) de Franz Kafka et dans Toute une histoire (1995) de Günter Grass », Germanica [En ligne], 31 | 2002, mis en ligne le 19 mars 2013, consulté le 06 octobre 2020. URL : http:// journals.openedition.org/germanica/2068; DOI : https://doi.org/10.4000/germanica.2068

Ce document a été généré automatiquement le 6 octobre 2020.

(c) Tous droits réservés 


\section{La réécriture de Bouvard et Pécuchet dans Un célibataire entre deux âges (1915), Recherches d'un chien (1922) de Franz Kafka et dans Toute une histoire (1995) de Günter Grass}

Die Umarbeitung von Bouvard et Pécuchet in Un célibataire entre deux âges (1915), Recherches d'un chien (1922) von Franz Kafka und in Toute une histoire (1995) von Günter Grass.

Florence Bancaud

1 Cette étude porte sur le dialogue fécond qui s'établit entre l'œuvre de Flaubert, Kafka et Grass : tous les trois ont le goût de la « réécriture qui modifie, c'est-à-dire, partant aussi d'un texte premier, accepte l'altération et tend vers l'altérité $»^{1}$. Chez ces auteurs qui incarnent la tension moderne entre respect de la tradition et volonté de renouvellement formel et thématique, la réécriture ne vise pas la fidélité au texte premier, mais son amélioration. Elle se donne donc comme "satisfaction d'une exigence virtuelle, réalisation d'un projet en train de s'élaborer $»^{2}$. Les œuvres de Kafka et Grass obéissent à la définition genettienne de l'hypertexte comme texte greffé, " texte dérivé d'un texte antérieur par transformation simple ou indirecte ${ }^{3}$ ou comme palimpseste « où l'on voit, sur le même parchemin, un texte se superposer à un autre qu'il ne dissimule pas tout à fait, mais qu'il laisse voir par transparence $»^{4}$. Mais le dialogue avec l'œuvre-référent s'accompagne autant du plaisir de répétition ou d'aliénation dans l'autre que du plaisir de dire autrement, qui suppose une pratique critique.

2 Pourquoi avoir choisi Bouvard et Pécuchet comme œuvre de référence? Parce que Flaubert considérait ce roman comme son testament littéraire où il entendait dénoncer l'universelle stupidité du genre humain. La genèse même du roman recouvre les dix- 
sept dernières années de sa vie. Des années marquées par des deuils, des déceptions, de graves difficultés financières et l'expérience d'une nation humiliée par la guerre de 1870. Flaubert, que la capitulation de Paris le 28 janvier 1871 et la Commune démoralisent, écrit alors à George Sand : "Je suis écoeuré, navré par la bêtise de mes compatriotes. L'irrémédiable barbarie de l'humanité m'emplit d'une tristesse noire $»^{5}$.

3 L'idée originelle du roman remonte en fait à avril 1863, date à laquelle Flaubert prévoyait d'écrire un récit intitulé les Deux cloportes. Le projet se précise le 1er juillet 1872 lorsque Flaubert informe Georges Sand de son projet d'écrire «une œuvre moderne qui aura aussi la prétention d'être comique ». La préparation commence véritablement en juillet 1872 et la rédaction en août 1874. Le roman exige des lectures effrayantes et épuise totalement Flaubert. Dès le 6 août 1874, il écrit en effet à sa nièce Caroline :

Je patauge, je rature, je me désespère. J'ai eu, hier soir, un violent mal d'estomac. Mais ça ira, il faut que ça aille. N'importe! Les difficultés de ce livre-là sont effroyables. Je suis capable d'y crever à la peine. L'important, c'est qu'il va m'occuper durant de longues années. Tant qu'on travaille, on ne songe point à son misérable individu ${ }^{6}$.

4 Dès 1875, Flaubert ne croit plus à son roman et le rejette. En avril, il est à bout de forces, épuisé et démoralisé par la matière même de ce « livre insensé » devenu pour lui un pensum. Rongé par l'amertume et le nihilisme, il décide d'interrompre la rédaction de son roman, déclarant : « Bouvard et Pécuchet étaient trop difficiles, j'y renonce... Quel gouffre que la bêtise humaine ! $\aleph^{7}$ Il délaisse son manuscrit pendant deux ans pour y revenir le 2 juin 1877. Malgré maints maux - insomnies, troubles oculaires, fracture du péroné -, il nage alors dans l'euphorie : « il est possible que je m'y noie, mais si je m'en tire, le globe terrestre ne sera pas digne de me porter... Quel bouquin! Ce formidable bouquin!». En 1879 il commence le dernier chapitre. Mais le 8 mai 1880, il meurt d'une hémorragie cérébrale, laissant un roman inachevé qui sera publié en 1881 et connaîtra un immense succès.

5 Cette œuvre centrale dans la vie de Flaubert constitue en elle-même un triple palimpseste. Flaubert y condense tout d'abord le fruit de son immense érudition héritée de sources aussi multiples qu'Appien, Pline, Procope, Diodore, Strabon, Pausanias, Hérodote, la Bible, le Pro Balbo de Cicéron... Du 20 septembre 1872 au 4 août 1873, il a absorbé 194 volumes; le 17 juin 1874, il a lu et résumé 294 volumes. 100 volumes de médecine donneront 16 pages, 3 volumes de botanique 6 lignes du roman. Mais cette voracité livresque est autant le signe d'un esprit encyclopédique que de la volonté de dénoncer le culte absolu de la référence : «Pour ce réquisitoire déguisé qui est aussi, dans la meilleure des hypothèses, une opération de délivrance, Flaubert ingurgite et rejette, ingère et s'exonère ${ }^{8}$."

Le roman constitue aussi la réécriture d'un texte antérieur. Flaubert se serait en effet inspiré d'une nouvelle de Barthélémy Maurice, Les deux greffiers, publiée dans la Gazette des tribunaux, dans le Journal des journaux en 1841 et dans l'Audience en 1858. Dans son Journal, Edmond de Goncourt dénoncera d'ailleurs le roman de Flaubert comme emprunt, sinon comme plagiat de cette histoire de deux employés qui décident de s'installer en Touraine avec leur femme où ils passent leur temps à chasser, à pêcher, à jardiner, puis, vaincus par l'ennui, se consacreront au travail de copie.

7 Ce roman peut enfin être lu comme un autopalimpseste de l'œuvre flaubertien. De fait, il y a déjà du Bouvard et Pécuchet dans Madame Bovary: comme le pharmacien d'Yonville, 
Bouvard et Pécuchet ont le goût des inscriptions, des étiquettes, du laboratoire, de lecture du journal et des débats sur les grandes questions. Mais ce sont des Homais qui doutent d'eux-mêmes et de la science. Ils rappellent aussi Charles Bovary, dont Bouvard porte d'ailleurs le prénom : ils sont aussi ignorants face au savoir que Charles devant ses cours de médecine et partagent le bovarysme, le désir d'être autre et ailleurs d'Emma.

Pourquoi rapprocher le roman de Flaubert des textes de Kafka et de Grass? Parce que, comme Bouvard et Pécuchet, ils retracent une quête de savoir et témoignent d'une soif de modèles qui finit par ôter toute originalité et toute individualité aux protagonistes. Ils constituent une autocaricature de l'écrivain, qui s'y pastiche souvent lui-même et tourne en ridicule le travail d'archiviste qui sous-tend ses romans. Enfin, ils sont empreints du pessimisme de leur auteur, témoin toujours sceptique de son temps et des contingences de l'histoire.

9 Comment la fascination de Kafka pour Flaubert s'explique-t-elle? Borgès a été un des rares à mettre en parallèle Bouvard et Pécuchet et l'œuvre de Kafka: "In the past, Bouvard et Pécuchet looks toward the parables of Voltaire, Swift and the orientals, in the future toward the parables of $\mathrm{Kafka}^{9}{ }{ }$. Flaubert a été l'écrivain le plus intensément et constamment admiré par Kafka, qui le définit, avec Grillparzer, Dostoïevski et Kleist, comme un de ses quatre "frères de sang ». Comme lui, Flaubert est de santé fragile, est victime de crises nerveuses qui compromettent tout travail pratique, et vit dans une ascèse littéraire absolue. Madame Bovary (1857), qu'il possède dans la traduction allemande, est son livre de chevet ; il a également lu l'Éducation sentimentale, Salambo, les Trois contes, La tentation, Bouvard et Pécuchet, la correspondance et les journaux. À propos de L'Éducation sentimentale, il écrit à Felice Bauer : "Il m'a fait tressaillir et il m'a pris totalement, et chaque fois je me suis senti comme un enfant spirituel de cet écrivain, encore que pauvre et maladroit. ${ }^{10}$ Comme Flaubert, Kafka prône l'exactitude narrative, l'effacement de l'écrivain derrière son œuvre et refuse l'analyse en profondeur des personnages, caractérisés par leurs seuls gestes et expressions. Tous deux dénient toute possibilité de liberté à l'homme, soumis au pouvoir du destin et qui fait à leurs yeux honte à son statut d'homo sapiens. Déplorant, comme Flaubert, la banalité du monde, Kafka voit en la littérature le seul salut possible. Rien d'étonnant à ce qu'il se réfère donc explicitement à Bouvard et Pécuchet en évoquant son récit Un célibataire entre deux âges :

Écrit un peu hier et aujourd'hui. Histoire du chien. Je viens de lire le début. C'est laid et cela provoque des maux de tête. En dépit de toute sa vérité, le récit est méchant, pédant, mécanique, c'est un poisson échoué sur un banc de sable et ne respirant plus qu'à peine. J'écris mon Bouvard et Pécuchet bien prématurément ${ }^{11}$.

10 Mais si on retrouve dans ce récit inachevé la figure du célibataire ascétique et le mépris flaubertien de l'humanité, c'est dans Recherches d'un chien que réapparaît le thème central de Bouvard et Pécuchet: l'échec du savoir encyclopédique et la solitude du chercheur englué dans des abstractions livresques ou des divagations de la pensée.

11 Quant à Grass, dont le maître absolu est Döblin, il se réfère à Flaubert comme à un des maitres du roman picaresque en qui il voit le seul genre qui ait pris une dimension européenne et dont il adopte la forme et les thèmes pour son roman, Toute une histoire :

Il me fallait trouver une forme de roman qui laisse libre cours à l'imagination. Donc pas un roman psychologique, pas un roman consacré à un conflit. Pas d'histoire d'amour triangulaire [...]. Dans mon dernier roman en date, qui met lui aussi en scène deux héros picaresques, Fonty et Hoftaller, j'ai aussi beaucoup été encouragé 
par les deux personnages principaux de Bouvard et Pécuchet, un roman qui utilise lui aussi une forme de récit picaresque ${ }^{12}$.

Ce qui fascine Grass dans Bouvard et Pécuchet est la satire des prétendus « hommes de science » et la remise en question du positivisme qu'ils incarnent :

Grass aura d'abord eu à l'esprit les déambulations de ces deux héros de Flaubert à travers une époque marquée par la Révolution de 1848, ainsi que leur «carrière " de gratte-papiers dans les administrations et leur insuccès en tant qu'hommes de lettres et de science [...] Les aventures de Bouvard et Pécuchet renvoient moins à des destinées individuelles [...] qu'à une problématique d'ensemble. Là encore, modèle picaresque et intention satirique convergent. Ne peut-on pas supposer que Flaubert a voulu marquer dans son roman la rupture d'avec les certitudes scientifiques $d u$ $\mathrm{XVIII}^{\mathrm{e}}$ siècle et son doute par rapport au positivisme de son propre siècle, en définitive que Flaubert considérait toute forme de savoir comme vouée à l'échec ? ${ }^{13}$

\section{L'intrigue des quatre récits}

13 Le but du roman de Flaubert est de livrer une encyclopédie de la bêtise humaine à travers les expériences et les échecs successifs de "deux imbéciles autodidactes ». Il s'agit de deux greffiers, Bouvard et Pécuchet, nés en 1791, qui, délivrés de leur profession par un héritage providentiel, se lient d'amitié en 1838 ; ils décident bientôt de quitter Paris pour se retirer dans la campagne de Chavignolles où ils se consacrent au savoir. Ils abordent une à une toutes les sciences et techniques: jardinage, agriculture, arboriculture, horticulture (ch. 2), chimie, médecine, hygiène, astronomie, zoologie, géologie (ch. 3), archéologie, histoire (ch. 4), littérature (ch. 5), politique (ch. 6 et 7), amour et hydrothérapie (ch. 8), religion (ch. 9), gymnastique, ésotérisme, éducation et utopies politiques (ch. 10).

Dans ce récit peu romanesque où l'intrigue ne joue plus un très grand rôle, la structure est toujours semblable: à chaque nouvelle tentative, menée sur le même modèle (documentation - expérimentation - évaluation), les deux apprentis-savants essuient un échec. Finalement découragés et lassés des prétentions excessives de la science, ils ne retrouvent leur joie de vivre qu'en revenant à leur première passion : la copie. Dans ce roman inachevé et complexe, qui présente des "excroissances monstrueuses et désordonnées ${ }^{14}$, le collage révèle l'éclatement du disparate et fait la démonstration de l'illusoire unité du savoir, de la discontinuité du temps et du pouvoir de la représentation subjective et du cliché :

L'aménagement d'une encyclopédie en roman. Comment ce lieu de rupture, cette fête de la discontinuité qu'est, par vocation, le dictionnaire, admettrait-il une « histoire continue? ${ }^{15}$

Flaubert y est en effet décidé à réduire au silence tous les raisonneurs et à « démontrer que l'éducation, quelle qu'elle soit, ne signifie pas grand' chose ».

Résumons à présent l'intrigue des récits de Kafka, qui empruntent à Flaubert la figure du célibataire pour le premier, la thématique des recherches ascétiques pour le second. Un célibataire entre deux âges, commencé en février 1915, est lui-même la réécriture de Le Malheur du célibataire, court récit de Kafka daté de 1913. Il stigmatise la solitude, la rigidité du célibataire et la dialectique esprit-corps très présente aussi dans le roman de Flaubert :

Il semble que ce soit affreux d'être célibataire et, vieillard gardant à grand'peine sa dignité, de demander accueil aux autres, quand on veut passer une soirée en compagnie; d'être malade, et, du coin de son lit, de considérer la chambre vide 
pendant des semaines; de toujours prendre congé à la porte des maisons, de ne jamais grimper l'escalier aux côtés de sa femme; de n'avoir dans sa chambre que des portes de communication s'ouvrant sur les appartements des autres; de rentrer chez soi en portant son repas du soir à la main ${ }^{16}$.

17 Le récit intitulé Un célibataire entre deux âges reprend deux ans plus tard cette thématique du célibataire endurci et devenu hostile à l'humanité qui l'entoure : il peut, comme Bouvard et Pécuchet, être défini comme un "livre des vengeances ». Il livre en effet le portrait grotesque d'un célibataire nommé Blumfeld, impropre à la vie conjugale, vigilant, épiant le moindre bruit, méfiant et cynique envers les enfants, détestant tout le monde : sa femme de ménage, son patron, ses stagiaires et surtout les enfants stupides comme le fils de la femme de ménage ou malins comme les petites filles du concierge, jugeant toute l'humanité stupide. Cet être misanthrope, maniaque, égoïste et méchant est une des pires caricatures que Kafka ait faites de lui-même. Les deux petites balles de celluloïd bruyantes et dérangeantes cristallisent les obsessions de Blumfeld et la résistance que le monde lui oppose, mais incarnent aussi les hallucinations et le délire de persécution d'un névrosé où Kafka se reconnaît lui-même. Il s'agit d'un récit empreint d'un comique grinçant et burlesque, expression d'un humanité laide et d'un monde misérable.

18 Le second récit, intitulé Recherches d'un chien, met également en scène une figure de célibataire solitaire et obsessionnel. Ce récit, rédigé en 1922 dans le douzième cahier du Journal, constitue une des plus longues nouvelles de Kafka. Il implique une réflexion sous-jacente sur le judaïsme - « chien » était l'insulte communément utilisée à l'époque de Kafka par les juifs occidentaux pour désigner les juifs de l'Est - ; mais le thème central est la recherche du chien sur l'origine de la nourriture. Au début du récit, le jeune chien - en qui on peut aisément reconnaître un double de Kafka - dit avoir un rapport problématique avec la race canine ; s'il aspire à la vie en commun, il éprouve un désir constant de solitude et de silence, conditions nécessaires à l'activité qu'il partage avec Bouvard et Pécuchet : la recherche. Il évoque tout d'abord sa rencontre avec les chiens musiciens dont la musique le fascine et l'épouvante à la fois. Puis il concentre ses recherches sur l'origine de la nourriture, la quête de la nourriture céleste et de la vérité. Puis intervient un changement soudain - qui fait écho à un texte écrit à la même époque par Kafka et intitulé Un champion de jeûne - : le chien choisit le jeûne contre la vie. Enfin, dans un ultime rebondissement, il rencontre un chien dont le chant lui annonce sa propre mort, qu'il accepte avec terreur, angoisse et honte, comme Joseph $\mathrm{K}$ à la fin du Procès. Le récit se clôt sur l'image de l'ultime libération du chien, voué désormais à la musique et qui a renoncé aux abstractions scientifiques par amour du monde et de la liberté. Dans ce récit tardif, Kafka se livre à la réécriture de son œuvre et de sa propre vie en partant du modèle flaubertien de la recherche ascétique du célibataire.

19 Dans Toute une histoire de Grass, qui fut souvent présenté comme le roman de la réunification allemande, on retrouve le modèle flaubertien du duo de deux protagonistes ou deux héros picaresques, Theodor Wuttke rebaptisé Fonty à cause de sa fascination pour Fontane, et Hoftaller, espion qui le suit et l'accompagne lors de ses pérégrinations dans le Berlin d'après la chute du mur. Ces deux inséparables se réfèrent constamment à l'histoire du xIxe siècle, notamment à la Révolution de 1848, événement-clé $\mathrm{du}$ récit flaubertien. Comme ce dernier, celui de Grass est un palimpseste : il est clairement désigné comme une réécriture de l'œuvre de Fontane, dont Fonty se dit le « continuateur », et du Tallhover de Hans-Joachim Schädlich. 


\section{Le décor des quatre récits} l'appartement de Blumfeld -, celui des romans de Flaubert et de Grass présente bien des similitudes. Tous deux s'ouvrent sur l'image des pérégrinations des deux protagonistes dans la rumeur de la grande ville, lieu de rencontre théâtralisé. Dans le Paris peint par Flaubert, le chaos ambiant se mêle à une impression de vide et de chaleur et le temps semble suspendu :

Au-delà du canal, entre les maisons que séparent des chantiers le grand ciel pur se découpait en plaques d'outremer, et sous la réverbération du soleil, les façades blanches, les toits d'ardoises, les quais de granit éblouissaient. Une rumeur confuse montait du loin dans l'atmosphère tiède; et tout semblait engourdi par le désœuvrement du dimanche et la tristesse des jours d'été ${ }^{17}$.

On retrouve cette impression de chaos et d'ennui dans le Berlin de la réunification peint par Grass :

Ils traversèrent un no man's land qui avait été désertique des dizaines d'années durant et dont à présent la vaste superficie n'attendait goulûment que des propriétaires. [...]

Çà et là, le Mur était déjà criblé de trous et révélait ses entrailles : des fers à béton qui s'encroûteraient bientôt de rouille. [...] « Le chaos! S'écria (Hoftaller). Ce n'est tout simplement que le chaos! $»^{18}$

Chez Flaubert comme Grass, la ville étouffante et la réalité quotidienne sont opposées à la fuite dans un monde plus ouvert. Chez Flaubert, le rêve de paradis bucolique se cristallise dans l'évasion dans la province de Chavignolles, où frappe l'immensité des champs; le jardin y figure métaphoriquement la quête d'un savoir encyclopédique foisonnant, qui assimile le monde à un livre et découpe la réalité au gré des disciplines répertoriées :

Deux allées principales, formant la croix, divisaient le jardin en quatre morceaux. Les légumes étaient compris dans les plates-bandes, où se dressaient, de place en place, des cyprès nains et des quenouilles [...] et une claire-voie, dans le fond, donnait sur la campagne ${ }^{19}$.

Chez Grass, le rêve de fuite en Angleterre échoue, mais la nostalgie des Cévennes françaises finit par ramener Fonty et sa petite-fille Madeleine dans cet univers naturel, où l'horizon s'éclaircit dans l'ultime image du roman :

Vers la mi-octobre arriva [...] la dernière carte postale. Elle disait tout: le côté brillant montrait un vaste paysage de collines, vert à l'avant, de plus en plus bleuté vers l'horizon [...]. Nous lûmes: «avec un peu de chance, nous nous retrouvons dans une région colossalement déserte [...]. Par temps stable, il est possible de voir loin $^{20}$.

Dans les Recherches d'un chien, le rêve de fuite est cristallisé dans la rencontre avec les chiens musiciens et aériens qui, par leur musique sublime, arrachent le chien à la vie ordinaire et au monde physique pour l'amener à remettre toutes les certitudes canines en question et à développer ses recherches métaphysiques. 


\section{La temporalité}

Tout le roman de Flaubert est placé sous le signe de la météorologie, acteur à part entière: le temps et, parallèlement, la conversation et la culture s'y dégradent progressivement. Le second vecteur de la temporalité est la linéarité de l'histoire, illustrée par de nombreuses dates comme 1839, 1840, 1848, 1851 et structurée par une chronologie interne marquée par des repères tels que "le lendemain », "la fin de la semaine ", « la veille du départ ». Comme Toute une histoire s'ouvre sur les images de la révolution de la chute du mur et de la réunification allemande, le roman flaubertien évoque la césure de la révolution de 1848 :

Dans la matinée du 25 février 1848, on apprit à Chavignolles, par un individu venant de Falaise, que Paris était couvert de barricades - et le lendemain, la proclamation de la République était affichée sur la mairie. Ce grand événement stupéfia les bourgeois ${ }^{21}$.

La durée totale du temps raconté y recouvre une trentaine d'années, mais la vie monotone et répétitive et le temps de l'attente s'opposent à l'événement ponctuel de l'expérience. L'écriture, répétitive et cyclique, se donne comme un collage de microrécits qui figurent les expériences et les échecs successifs et obéissent à un schéma toujours identique : un événement provoque une idée, laquelle suscite la recherche documentaire qui débouche sur une expérience; cette dernière est gênée par des obstacles et se traduit par un résultat négatif; enfin, un "joint» relance l'enthousiasme pour une nouvelle recherche.

Dans les Recherches d'un chien, on retrouve cette succession de recherches n'aboutissant à aucun résultat probant et donnant au lecteur l'impression d'une temporalité figée. Dans Toute une histoire, le récit obéit à un mouvement de balancier entre le passé du XIxe siècle - incarné par la vie et l'œuvre de Fontane, la révolution de 1848 et l'empire de Bismarck -, le présent de la réunification allemande et le futur incarné par Madeleine, le petite-fille de Fonty. Mais on y observe aussi une structure cyclique et répétitive - figurée entre autres par les métaphores du paternoster, et du grèbe huppé qui dénonce l'incapacité foncière de l'homme à tirer des leçons de l'histoire: Fonty, désireux de « prendre en charge » et de perpétuer la mémoire de Fontane, réplique ses expériences d'adultère, ses crises nerveuses, ses promenades, partage ses goûts et ses tics de langage. De même, la seconde unité allemande est dénoncée comme une possible réplique des échecs et dangers liés à l'impérialisme allemand de la première unité, qui a «bousillé la démocratie » et a donné naissance au Troisième Reich : le 18 janvier 1990 est le jour anniversaire de la fondation de l'Empire en 1871; la Treuhand sera logée dans la Maison des Ministères qui a habité le ministère de l'aviation du troisième Reich.

Dans tous ces récits, la structure temporelle est donc cyclique : si elle dénonce l'éternel retour du même dans l'histoire, elle stigmatise surtout l'incapacité de l'homme à tirer des leçons de l'histoire et à réadapter sa théorie et sa pratique à une réalité qui lui reste toujours étrangère et insaisissable.

\section{Le ridicule des personnages}

Dans le roman de Flaubert et de Grass, le grotesque des personnages naît de l'association de contraires à la fois incompatibles et complémentaires. Dès l'instant où ils convergent Boulevard Bourdon, on sent Bouvard et Pécuchet promis l'un à l'autre, 
mais leurs différences sautent immédiatement aux yeux : l'un est bedonnant, l'autre filiforme, l'un court les dames, l'autre les ignore. L'un est prodigue, l'autre économe. Ils ne marchent, ne s'habillent, ne rient ni ne calligraphient de la même façon. Leurs goûts mêmes sont antinomiques. À Chavignolles, Bouvard s'avère être un matérialiste pragmatique, un aristotélicien, un neptunien constitutionnel tandis que Pécuchet est un pur spiritualiste platonicien, un plutonien, un sans-culotte. Ces différences se retrouvent jusque dans leurs prénoms :

Il y a dans Bouvard du bavard et du boute-en-train, du bedonnant et du concupiscent : et dans Pécuchet dont le syllabes sonnent comme une triple croche, de la maigreur et de la retenue, un côté vieille fille, un chut !22

On retrouve chez Grass cette association de contraires : à l'imagination fertile et à la tendance contemplative de Fonty-Wuttke s'oppose le pragmatisme de Hoftaller, son double, son « ombre diurne et nocturne » si différente de lui et pourtant indispensable à son équilibre. Dans les deux romans, l'harmonie née du contraste fait tout le grotesque de la description liminaire et on note l'étonnante parenté des deux portraits de ces hommes en mouvement dans l'immensité de la ville qui les entoure et les écrase :

\begin{tabular}{|c|c|c|}
\hline MOTIFS & & GRASS \\
\hline $\begin{array}{l}\text { - Motif de } \\
\text { l'apparition : }\end{array}$ & FLAUBERT & $\begin{array}{l}\text { Voici que résolument ils apparurent } \\
{[\ldots]:}\end{array}$ \\
\hline $\begin{array}{l}\text { - Différence de } \\
\text { taille et de } \\
\text { corpulence: } \\
\text { - Motifs du } \\
\text { chapeau et du } \\
\text { manteau : }\end{array}$ & $\begin{array}{l}\text { Deux hommes parurent. L'un venait de } \\
\text { la Bastille, l'autre du Jardin des Plantes. } \\
\text { Le plus grand, vêtu de toile, marchait le } \\
\text { chapeau en arrière, le gilet déboutonné } \\
\text { et sa cravate à la main. Le plus petit, } \\
\text { dont le corps disparaissait dans une } \\
\text { redingote marron, baissait la tête sous } \\
\text { une casquette visière pointue. Quand ils } \\
\text { furent arrivés au milieu du boulevard, } \\
\text { ils s'assirent à la même minute, sur le } \\
\text { même banc. [...]. } \\
\text { L'aspect aimable de Bouvard charma } \\
\text { tout de suite Pécuchet. [...] et ses } \\
\text { cheveux blonds, frisés [...] lui } \\
\text { donnaient quelque chose d'enfantin. } \\
\text { [...]. L'air sérieux de Pécuchet frappa } \\
\text { Bouvard [...] les mèches garnissant son } \\
\text { crâne élevé étaient plates et noires.... }\end{array}$ & $\begin{array}{l}\text { long et mince l'un, trapu et costaud } \\
\text { l'autre. La silhouette de leurs chapeaux } \\
\text { et manteaux, feutre noir, laine } \\
\text { mélangée grise, se fondait en un tout } \\
\text { qui allait grandissant. [...] Tantôt } \\
\text { gesticulait la moitié élancée, tantôt la } \\
\text { râblée [...]. Vus depuis la porte de } \\
\text { Brandebourg, ils devinrent de plus en } \\
\text { plus petits. Le couple inégal. [...] Le film } \\
\text { muet. L'un marchant à grands pas, } \\
\text { l'autre trottinant }{ }^{24} \text {. }\end{array}$ \\
\hline $\begin{array}{l}\text { - Motif du } \\
\text { contraste des } \\
\text { êtres } \\
\text { contraires: }\end{array}$ & & \\
\hline
\end{tabular}

31 Comme le souligne Roger Kempf ${ }^{25}$, l'association des contraires n'est pas seulement emblème de fraternité, mais figure originelle, initiatrice d'entreprises et de discours ; 
dans le désordre s'improvise une encyclopédie dont les articles se nomment : ivrogne, ouvriers, noce, femmes, célibat, solitude, fille de joie, soldat, petite vérole, militaire, religion, ponts et chaussées, régie des tabacs, commerce, théâtre, marine, au restaurant: épices, sciences, gagne-pain, Au café, becs de gaz, luxe, journaux. La digression est ici le principe du discours et de la relation des protagonistes :

Leurs paroles coulaient intarissablement, les remarques succédant aux anecdotes, les aperçus philosophiques aux considérations individuelles. Ils dénigrèrent le corps des Ponts et Chaussées, la régie des tabacs, le commerce, les théâtres, notre marine et tout le genre humain, comme des gens qui ont subi de grands déboires. Chacun en écoutant l'autre retrouvait des parties de lui-même oubliées ${ }^{26}$.

De même, c'est le ton de la causerie qui est résolument adopté par Fonty-Wuttke et Hoftaller lorsqu'ils se promènent dans les rues de Berlin, évoquant tantôt le passé sentimental de Fonty-Wuttke, tantôt la première unité allemande, tantôt la réunification et ses conséquences comme la réforme monétaire ou les privatisations de la Treuhand, tantôt leur conception du rôle de l'écrivain dans la société. Anecdotes, pensées, réflexions, citations, proverbes sont ainsi collés dans un puzzle narratif dont les éléments disparates témoignent des contradictions et des paradoxes du réel.

\section{Une identité problématique}

Ce qui frappe chez les protagonistes de Flaubert et de Grass est leur absence d'individualité. Chez Flaubert, elle s'explique par une ascendance déficiente - Pécuchet n'a pas connu sa mère, Bouvard est né bâtard - et par leur fascination pour la copie. Chez Grass, l'absence d'individualité naît aussi de la référence constante à des modèles dont les héros sont les ombres ou les doubles. Fonty-Wuttke est le sosie de Fontane : « les traits de Fonty, en face comme de profil, étaient ceux de l'Immortel " ${ }^{27}$. S'il assume sa ressemblance avec Fontane jusqu'à s'habiller comme lui - «l'écharpe faisait partie de la survivance de l'original $»^{28}$-, à adopter ses tics de langage comme l'emploi fréquent des adjectifs «colossal » et « ridicule » et à citer ou méditer en permanence des passages des œuvres de Fontane, Hoftaller frappe par l'aspect peu marquant de ses traits et «sa façon de passer tellement inaperçu qu'on le remarquait. ${ }^{29}$ C'est cette absence d'individualité qui fait de lui à la fois l'ombre de Tallhover et de Fonty:

Le physique de Hoftaller n'est attesté par aucune photo, et encore moins par un portrait dessiné. Et comme le biographe de Tallhover ne nous a rien fourni d'utilisable, il ne nous reste qu'à espérer qu'avec Fonty tout entier entre aussi dans le champ, au moins vaguement, son ombre diurne et nocturne ${ }^{30}$.

Chez nos quatre protagonistes, la multiplication de professions en fait autant de masques destinés à voiler une identité problématique. Pécuchet a, depuis l'âge de 15 ans, tâté de tous les emplois : maître d'études, élève en pharmacie, comptable navigant ou "copiste au ministère de la marine ». Bouvard a tenu une boutique de confiseur, puis, après six mois de mariage, vu sa femme s'envoler avec la caisse et à été condamné à retourner au célibat pour travailler enfin comme copiste dans une maison de commerce. Quant à Hoftaller et Fonty-Wuttke, ils ont travaillé sous le régime nazi, puis pour l'État des ouvriers et paysans de RDA, et enfin comme espion de la Stasi dans le cas d'Hoftaller. Ils sont donc devenus experts dans l'art de "retourner leur veste » et de s'adapter aux changements de système, comme Bouvard et Pécuchet se réadaptent à chaque nouveau système qu'ils expérimentent et comme le chien des Recherches se 
contraint à un questionnement toujours nouveau, finissant par avouer: "Avec les questions, c'est moi seul que je poursuis, je veux me stimuler au moyen du silence $»^{31}$.

\section{Les rapports complexes avec les autres hommes}

Tous nos récits mettent en scène des personnages solitaires, distants par rapport à l'humanité qui les entoure et envers laquelle ils s'avèrent tantôt méprisants, tantôt en quête de communauté. Ainsi le chien des Recherches déclare-t-il :

Retiré, solitaire, occupé seulement de mes petites recherches, des recherches menées sans espoir, mais qui me sont indispensables, c'est ainsi que je vis ; mais, même de loin, je continue à avoir l'œil sur mon peuple ; [...] à l'occasion, je leur donne aussi de mes nouvelles. On me traite avec respect; on ne comprend pas ma manière de vivre, mais on ne m'en tient pas grief. [...] On ne doit pas perdre de vue, en effet, que, malgré mes singularités évidentes, je suis bien loin d'avoir renié ma race $^{32}$.

On retrouve cette image du chien dans Un célibataire entre deux âges où Blumfeld, pour tromper sa solitude, désire avoir un chien, métaphore de l'« autre-juif » pour Kafka :

Blumfeld veut seulement un compagnon, une bête dont il n'ait pas beaucoup à s'occuper [...], mais qui soit aussi à sa disposition, dès que Blumfeld en a envie, pour aboyer, sauter sur lui et lui lécher les mains ${ }^{33}$.

Mais ce désir de communauté cache aussi un profond mépris de Blumfeld pour l'humanité qui l'entoure : il déplore la laideur de sa femme de ménage et la stupidité de son petit garçon, et, dans l'usine de lingerie où il est employé, tient sous ses ordres deux stagiaires qu'il surveille et harcèle en permanence.

Les romans de Flaubert et Grass mettent en scène deux compagnons liés non seulement par un respect réciproque et une forme de solidarité existentielle, mais aussi par leur profond scepticisme envers l'humanité qui les entoure. Fonty et Hoftaller assistent avec distance aux manifestations de joie pour l'unité allemande et clament en permanence leur mépris pour ceux qui se laissent aller comme des marionnettes au gré du vent de l'histoire. Ainsi Fonty déplore-t-il l'enthousiasme naïf de la fête de l'unité où il voit une pure fiction et où il sent pointer le germe de la division : « Ils n'ont pas la moindre idée de ce que c'est que l'unité, mais ils font la fête. $»^{34}$ Bouvard et Pécuchet fustigent également l'esprit de troupeau de leurs semblables. Étudiant la question du suffrage universel, Bouvard déclare avec un scepticisme que lui reprochera Pécuchet :

Je crois plutôt à la bêtise du peuple. Ces nigauds forment la masse électorale, et nous subissons leur volonté. [...] De même, par le seul fait de la foule, les germes qu'elle contient se développent et il en résulte des effets incalculables ${ }^{35}$.

"Dégoûtés du petit nombre comme du grand ", ils sont convaincus que «la plèbe, en somme, valait l'aristocratie $»^{36}$ : ils méprisent le peuple autant que le bourgeois qui incarne l'ordre établi, le matérialisme et le conformisme ; ils critiquent tout autant les notables comme le médecin ou le notaire que les aristocrates qui prétendent incarnent la grandeur et l'autorité morale. Dans ce qui aurait dû devenir le second livre, Flaubert projetait de leur faire rassembler pour le Dictionnaire des Idées reçues les idées absurdes de leurs contemporains, et de faire d'eux les instruments d'une satire en règle de la société et de l'humanité

Mais ce rapport ambigu avec la société vient aussi de la marginalisation dont sont victimes ces personnages souvent critiqués par leurs contemporains ou en proie à leur 
moquerie. Dans Toute une histoire, les fils de Fonty se détournent de lui tandis que sa fille déplore son identification abusive avec Fontane. Chez Kafka, le personnage central est toujours un homme seul face à l'humanité environnante, qui soit ne le comprend pas comme dans les Recherches, soit le craint et le fuit, comme Blumfeld. Dans le roman de Flaubert, la quête de reconnaissance sociale est également condamnée à l'échec. Visitant le jardin de Bouvard et Pécuchet, leurs hôtes sont pris d'un fou-rire :

Madame Bordin éclata de rire. Tous firent comme elle. Le curé poussait une sorte de gloussement, Hurel toussait, le Docteur en pleurait, sa femme fut prise d'un spasme nerveux ${ }^{37}$.

On critique ensuite leurs expériences en matière de médecine, leurs opinions politiques et religieuses et leur système d'éducation, qui remettent en cause l'ordre établi :

On dédaigna leur système. Ils en firent un article pour le journal de Bayeux, une note au Préfet, une pétition aux Cambres, un mémoire à l'empereur. Le journal n'inséra pas leur article; le Préfet ne daigna répondre; les chambres furent muettes, et ils attendirent longtemps un pli du Château. [...] Bref, ils devenaient incommodes $^{38}$.

Dans toutes les œuvres de notre corpus, les rapports avec les femmes sont des plus problématiques : les deux célibataires de Kafka sont aigris et solitaires et chez Grass et Flaubert, les femmes sont perçues comme des éléments perturbateurs. Bouvard tente en vain de demander en mariage Madame Bordin et Pécuchet hérite de sa relation clandestine avec la petite bonne une maladie secrète. Tous deux décident finalement de renoncer totalement aux femmes :

Étrange besoin, est-ce un besoin ? Elles poussent au crime, à l'héroïsme et à l'abrutissement ! L'enfer sous un jupon, le paradis dans un baiser. [...] C'était le désir d'en avoir qui avait suspendu leur amitié. Un remords les prit. Plus de femmes, n'est-ce pas ? Vivons sans elles! Et ils s'embrassèrent avec attendrissement ${ }^{39}$.

Dans Toute une histoire, l'impossibilité d'entretenir avec les femmes un rapport de confiance et d'égalité se cristallise dans la thématique - empruntée à Fontane - de l'union impossible avec l'être aimé. Ainsi Fonty a-t-il quitté Madeleine Blondin, la maitresse rencontrée pendant la guerre, dans les Cévennes. Il souffre également d'une union difficile avec une épouse manquant totalement de compréhension pour lui et lui reprochant son mimétisme envers Fontane, son ambition politique et son manque de considération pour les siens, ce qui l'amène à résumer sa vie conjugale comme une "guerre de Trente ans $»^{40}$. La solution trouvée est le retrait ou le repli sur soi chez Flaubert et Kafka. Chez Grass, c'est la fuite dans l'imaginaire et la fuite réelle en France.

\section{La satire du savoir livresque et des abstractions encyclopédiques}

Les personnages de Flaubert, Grass et Kafka sont tous aux prises avec le monde et sa représentation livresque, scientifique ou religieuse. En 1872, Flaubert définit son roman comme "l'histoire de deux bonshommes qui copient une espèce d'encyclopédie critique en farce ». Bouvard et Pécuchet peut de fait se lire comme un "vaste roman d'apprentissage négatif qui sacralise et désacralise en même temps le savoir en le transformant en objet inaccessible, lieu d'un manque et d'un désir sans cesse frustré " ${ }^{41}$. On y assiste à l'oscillation constante entre vérité et erreur, savoir et ignorance, désir et frustration. 

principe de redondance dénonce l'impossibilité : redondance de citations de Fontane chez Grass, redondance d'expériences infructueuses chez Flaubert, redondance de questions du chien kafkéen. Le principe de tous ces protagonistes étant la copie, il ne peut être qu'infructueux : «Les personnages s'abolissent dans le mouvement même de leur copie $»^{44}$, mode de redondance stérile par excellence selon Foucault, pour qui « copier c'est ne rien faire ; c'est être les livres que l'on copie, c'est être cette infime distension du langage qui se redouble, c'est être le pli du discours sur lui-même ${ }^{45}$." Tous les protagonistes finissent par s'engluer dans une abstraction qui les détourne de la vie et les conduit à une ascèse dont les effets désastreux sur le corps se traduisent par la maladie des personnages. L'excès de lectures finit par «ébranler la cervelle ${ }^{46} \mathrm{de}$ Bouvard et Pécuchet, dont la santé fragile se manifeste par une jaunisse et dont le médecin souligne ironiquement la faiblesse et l'irritabilité : «trop de nerfs, trop artiste! $»^{47}$. Fonty sombre quant à lui dans de fréquentes crises nerveuses dont il ne parvient à guérir que par l'écriture de ses souvenirs d'enfance; quant au chien des Recherches, il pousse l'ascèse jusqu'au jeûne qui, loin de lui révéler la vérité qu'il recherche, témoigne de son abandon et son échec. À force de les amener à s'abîmer dans les livres et les recherches, la science finit donc par détourner de la vie et à figer dans l'abstraction. Le chien kafkéen déclare : « la science est riche en savoir, mais assez pauvre en succès pratiques $»^{48} ;$ «si seulement on pouvait rendre ce savoir efficace; si seulement on pouvait l'amener au grand jour! »" ; «À quoi bon les questions? J'ai échoué avec elles. $\aleph^{50}$. Tous ces récits se rejoignent donc dans la dénonciation de l'esprit de système initiée par Flaubert :

Ce livre touche à ce qu'il y a de plus grand, de plus curieux, de plus subtil et de plus intéressant dans l'homme: c'est l'histoire de l'idée sous toutes ses formes, dans toutes ses manifestations, avec toutes ses transformations, dans sa faiblesse et dans sa puissance [...]. Dans Bouvard et Pécuchet, les véritables personnages sont des systèmes et non plus des hommes. Les acteurs servent uniquement de porte-voix aux idées qui, comme des êtres se meuvent, se joignent, se combattent et se détruisent ${ }^{51}$.

Ce n'est donc pas le pouvoir bienfaisant, mais la force destructrice de la pensée qui se voit dénoncée. Dans la continuité du diagnostic prophétique émis par Balzac dans la préface de la Comédie humaine - "Si la pensée est l'élément social, elle est aussi l'élément destructeur »-, les trois récits dénoncent le danger de l'intoxication par la littérature et la pensée et fustigent l'exaspération forcenée de la vie consciente où ils voient le mal de la civilisation moderne. Flaubert s'inscrit dans le débat 
épistémologique de son siècle, Kafka dénonce le pouvoir morbide de la réflexion et Grass mène une réflexion critique sur l'identification romanesque et sur une confusion entre réalité et fiction qui atteint son point culminant lors de l'incendie de la Treuhand anticipé dans le discours enflammé de Fonty.

Si la trame narrative des trois récits est si répétitive, c'est que la forme subversive de ces récits où l'intrigue s'éparpille dans la répétition comique des expériences tend à faire des personnages des marionnettes dérisoires et tragiquement déchirées entre leur soif d'absolu et la vanité de leurs efforts. La circularité de leur parcours fait que le texte ne peut être qu'infini ; mais cet inachèvement, loin de figurer un absolu inaccessible, témoigne de l'impossibilité d'atteindre la totalité, l'absolu, la vérité et ouvre l'abîme béant du scepticisme :

Tout est variation, différence, relativité (sauf l'échec) : les bienséances, les dogmes. Pas de point de vue absolu sur les choses [...], mais des visées partielles, locales, subjectives, limitées dans le temps et l'espace ${ }^{52}$.

50 Selon Peter Bürger, la seule certitude de Flaubert, suivi par Kafka et Grass, serait donc qu'il n'y a rien à raconter, et que « c'est précisément cela, la seule chose digne d'être racontée... $»^{53}$ :

La complexité du monde moderne, dans lequel les individus ne sont plus porteurs de grands projets, mais de petits actes égoïstes, va à l'encontre d'une esthétique qui aspire à la grandeur et à la simplicité de l'effet. «la beauté n'est pas compatible avec la vie moderne». [...] Comment peut-on raconter s'il n'y a plus rien à raconter? Que peut-on mettre dans un roman si plus rien n'émerge de la grisaille de la vie quotidienne, et s'il n'y a plus de grandes actions ni de grandes passions? Le protagoniste de ce roman sera l'individu moyen, Monsieur tout le monde, ses actes des non-actes, mouvement vide de l'ici et là ${ }^{54}$.

51 Flaubert, Kafka et Grass mettent en scène l'atrophie de l'expérience dans une société moderne d'individus atomisés et désindividualisés ainsi que le désenchantement du monde stigmatisé par Weber, pour qui l'intellectualisation et la rationalisation croissantes ne signifient pas «un accroissement général de notre connaissance concernant les conditions de vie dans lesquelles nous sommes. [...] Mais cela signifie : le désenchantement du monde. ${ }^{55} \mathrm{Si}$ ces quatre récits retracent un échec de l'apprentissage des héros perpétuellement tendus vers une vérité qui se dérobe, leur seule force reste l'ironie amère qui, elle, joue un rôle éducatif en révélant les dangers du dogmatisme :

Vouée à débouter les fausses valeurs, l'écriture choisit de piétiner dans son scepticisme, par crainte de sombrer elle-même du côté du dogmatisme [...]. Quand le langage est un chaudron fêlé, l'écriture ne saurait posséder un pouvoir affirmatif. [...]. Il ne reste plus alors effectivement, en deuil de la vérité, que le plaisir de l'ironie, langage nihiliste, langage au second degré, langage de la mention ${ }^{56}$.

Témoignant des limites de l'esprit humain et de l'impossible quête de vérité et d'absolu dans le monde moderne, ces récits peuvent bien sûr être lus comme les récits d'une formation ratée. Bouvard et Pécuchet se résignent à rester des copistes, Fonty et Hoftaller se séparent et le voyage de Fonty en France ressemble fort à une fuite ; enfin, le chien des Recherches avoue posséder un instinct qui a détruit en lui les aptitudes scientifiques et l'a ramené vers le monde humain. Mais c'est cet instinct de survie et cette volonté d'aller de l'avant qui restent la force ultime de tous ces êtres certes limités, mais qui tiennent leur dynamique du lien qu'ils conservent avec le monde et de la compagnie d'autrui : le compère copiste chez Flaubert, la petite-fille de Fonty chez Grass, le chasseur et les congénères-chiens chez Kafka. Si ces récits ne peuvent plus 
être définis comme des récits de formation, c'est donc parce qu'ils ne prétendent plus livrer de message au lecteur; tout au plus suscitent-ils en lui, et c'est là ce qui fonde tout leur prix et leur modernité, l'intuition que c'est dans le mouvement de la recherche et dans le rejet de l'esprit de système que doit résider la force et la dignité de l'homme, ce Sisyphe moderne :

Persuadé de l'origine tout humaine de ce qui est humain, aveugle qui désire voir et qui sait que la nuit n'a pas de fin, il est toujours en marche. Le rocher roule encore [...]. [...]. La lutte elle-même vers les sommets suffit à remplir un cœur d'homme. Il faut imaginer Sisyphe heureux ${ }^{57}$.

\section{NOTES}

1. La Réécriture du texte littéraire, GRELIS, Annales littéraires de L'université de Besançon, Paris, Les Belles Lettres, 1987, p. 15.

2. Ibid., p. 15.

3. Gérard Genette, Palimpsestes. La littérature au second degré, Paris, Seuil, 1982, p. 8.

4. Ibid., p. 556.

5. Flaubert, Lettre à Georges Sand, 22 juillet 1870, Correspondance, t. IV, Paris, Gallimard (Bibliothèque de La Pléiade), 1998, p. 211.

6. Ibid., À sa nièce Caroline, 6 août 1874, p. 847.

7. Ibid., À Edma Roger des Genettes, 3 octobre 1875, p. 969-970.

8. Roger Kempf : Bouvard, Flaubert et Pécuchet, Paris, Grasset, 1990, p. 38.

9. Borges, cité in Charles Bernheimer, Flaubert and Kafka. Studies in psychopoetic structure, New Haeven and London, Yale university press, 1942, p. 102.

10. Kafka, Lettre à Felice, 15 novembre 1912, in: Euvres Complètes IV, Paris, Gallimard (Bibliothèque de La Pléiade), 1989, p. 60.

11. Journal, 9 février 1915, p. 383.

12. Günter Grass, interview avec Olivier Mannoni, Magazine littéraire ${ }^{\circ} 381$, novembre 1999, p. 22.

13. Philippe Wellnitz, «Ein weites Feld, un roman picaresque? », in Günter Grass, Ein weites Feld / Toute une histoire, Strasbourg, Presses Universitaires de Strasbourg, 2001 p. 187.

14. Thanh-Vân Ton-Thât, Lectures d'une cuvre. Bouvard et Pécuchet, une odyssée de la bêtise, Paris, Éditions du Temps, 1999, p. 36.

15. Kempf, op. cit., p. 43.

16. Kafka, Le Malheur du célibataire, in : CEuvres Complètes II, Récits, Paris, Gallimard (Bibliothèque de La Pléiade), 1980, p. 170.

17. Flaubert, Bouvard et Pécuchet, Paris, Gallimard, 1979, p. 51.

18. Grass, Toute une histoire, Paris, Seuil, 1997, p. 15-17.

19. Bouvard et Pécuchet, p. 74.

20. Toute une histoire, p. 723.

21. Bouvard et Pécuchet, p. 226.

22. Roger Kempf, op. cit., p. 82.

23. Bouvard et Pécuchet, p. 52.

24. Toute une histoire, p. 15622.

25. Roger Kempf, op. cit., p. 69-70. 
26. Bouvard et Pécuchet, p. 54.

27. Toute une histoire, p. 44.

28. Ibid., p. 49.

29. Ibid., p. 49.

30. Ibid., p. 47

31. Recherches d'un chien, p. 688.

32. Recherches d'un chien, p. 675.

33. Un célibataire entre deux âges, p. 357.

34. Toute une histoire, p. 419.

35. Bouvard et Pécuchet, p. 242.

36. Ibid., p. 243.

37. Bouvard et Pécuchet, p. 109.

38. Ibid., p. 405.

39. Ibid., p. 271-272.

40. Toute une histoire, p. 182.

41. Ibid., p. 77.

42. Recherches d'un chien, p. 683.

43. Ibid., p. 689.

44. Stéphanie Dord-Crouslé, p. 102.

45. Michel Foucault, «la bibliothèque fantastique", in: Travail de Flaubert, collectif, sous la direction de G. Genette et T. Todorov, Paris, Ed. du Seuil (Points Littérature), 1983, p. 121-122.

46. Bouvard et Pécuchet, p. 134.

47. Ibid., p. 211.

48. Recherches d'un chien, p. 685.

49. Ibid., p. 686.

50. Ibid., p. 694.

51. Guy de Maupassant, Étude sur Gustave Flaubert (1884), repris dans Guy de Maupassant, Pour Gustave Flaubert, Paris, Éditions Complexe, « le regard littéraire », 1986, p. 55-58.

52. Yvan Leclerc, La Spirale et le monument. Essai sur Bouvard et Pécuchet de Gustave Flaubert, Paris, Sedes, 1988, p. 90.

53. Peter Bürger, op. cit., p. 254-255.

54. Peter Burger, La Prose de la modernité, p. 252-253.

55. Max Weber, «Vom inneren Beruf zur Wissenschaft », in: Soziologie, Universalgeschichtliche Analysen, Politik, Ed. Winckelmann, Stuttgart, Kröner, 1973, S. 317.

56. Philippe Dufour, Flaubert et le pignouf. Essai sur la représentation romanesque du langage, Paris, Presses Universitaires de Vincennes, 1993, p. 185-186.

57. Albert Camus, Le Mythe de Sisyphe, Paris, Gallimard (coll. Folio), 1942, p. 163.

\section{RÉSUMÉS}

Cette étude porte sur la réécriture de Bouvard et Pécuchet, le testament littéraire de Flaubert, dans Un célibataire entre deux âges (1915) et Recherches d'un chien (1922) de Kafka et Toute une histoire (1995) de Grass. Si le premier récit de Kafka ne reprend que la figure du célibataire aigri et la thématique du mépris de ses contemporains, les Recherches et Toute une histoire frappent par leurs 
nombreuses similitudes avec le roman de Flaubert. Le thème central de ces récits, articulés autour du thème de l'errance physique et métaphysique, et structurés par une temporalité cyclique, est la quête de l'identité et de la vérité, soit par la recherche (chez Flaubert et Kafka), soit par la perpétuation de modèles (chez Grass). Mais tous se soldent par l'échec de la formation des protagonistes et dénoncent le manque d'identité de ces Don Quichotte modernes, abîmés dans le mouvement de la copie, englués dans les abstractions livresques et inaptes à s'accommoder d'une humanité qu'ils contemplent avec scepticisme et dont ils sont souvent méprisés ou incompris. Tous ces récits se rejoignent donc dans la démonstration du désenchantement du monde moderne, où les notions de beauté, d'individualité, de vérité, de totalité, de récit même disparaissent au profit de la peinture de la grisaille et de l'aliénation quotidiennes, du scepticisme et de la relativisation de toutes les valeurs, dont ne subsiste que ce qui demeure la dernière liberté et dignité humaine : le questionnement et la quête constante d'un sens certes virtuel, mais dont toute la force réside dans le désir et la dynamique qu'il suscite.

Folgende Studie untersucht die Umarbeitung von Bouvard und Pécuchet, Flauberts literarischem Testament, in Kafkas Ein älterer Junggeselle (1915) und Forschungen eines Hundes (1922) und Grass' Ein weites Feld (1995). Während Kafkas erste Erzählung nur die Figur des Junggesellen und die Thematik der Verachtung der Mitmenschen wiederaufnimmt, fallen zahlreiche Parallelen zwischen Flauberts Roman und den Forschungen sowie ein weites Feld auf. Das zentrale Thema dieser Erzählungen, die um das Thema der physischen und metaphysischen Heimatlosigkeit und der Sinnsuche kreisen, und die durch eine zyklische Zeit strukturiert werden, ist die Identitätsund Wahrheitssuche, entweder durch die Forschung (bei Flaubert und Kafka) oder durch die Verewigung gewisser Modelle (bei Grass). Alle enden aber mit dem Scheitern der Bildung der Protagonisten und entpuppen die mangelnde Identität jener modernen Don Quichotten, die in eine mimetische Geste versinken, in Bücherabstraktionen verharren und unfähig sind, sich mit einer Menschheit abzufinden, welche sie skeptisch beobachten oder von welcher sie oft verachtet oder missverstanden werden. Alle Erzählungen demonstrieren also auf ähnliche Weise die Entzauberung der modernen Welt, wo solche Begriffe wie Schönheit, Individualität, Wahrheit, Totalität, ja sogar Erzählstoff zugunsten der Schilderung des grauen Alltags, der Entfremdung, der Skepsis und der Relativierung aller Werte verschwinden. Was davon übrig bleibt, ist das einzige Zeichen der menschlichen Freiheit und Würde : das Fragen und das ständige Suchen nach einem zwar virtuellen Sinn, dessen ganze Kraft aber in dem Streben und in der Dynamik liegt, die es bewirkt.

\section{AUTEUR}

\section{FLORENCE BANCAUD}

Université de Rouen 